\title{
Stellar Feedback Through Cosmic Time: Starbursts \& Superwinds
}

\author{
Michael A. Dopita \\ Research School of Astronomy \& Astrophysics, The Australian National University \\ Cotter Road, Weston Creek, ACT 2611, Australia \\ email: Michael.Dopita@anu.edu.au
}

\begin{abstract}
Throughout cosmic time, the feedback of massive star winds and supernova explosions has been instrumental in determining the phase structure of the interstellar medium, controlling important aspects of both the formation and evolution of galaxies, producing galactic winds and enriching the intergalactic medium with heavy elements. In this paper, I review progress made in our theoretical understanding of how these feedback processes have operated throughout cosmic time from the epoch of the first stars through to the present day.
\end{abstract}

Keywords. galaxies: evolution - galaxies: high-redshift - intergalactic medium - ISM: evolution - ISM: jets and outflows - stars: winds, outflows

\section{Introduction}

Feedback from massive stars exercises an important control on a wide number of aspects of the evolution of our Universe. Specifically, the formation of massive stars controls the re-ionization of the Universe at the termination of the "dark age". The ionization of the intergalactic gas radically alters the development of structure in the Universe and the subsequent collapse of galaxies. Once galaxies have been formed, the energy and ionization feedback of massive stars determines the phase structure of the interstellar medium in galaxies, and controls structural parameters of disk galaxies such as the scale height of the gaseous component. In starburst regions, the over-pressure can encourage collapse of molecular clouds, triggering new generations of stars to form. This over-pressure can in turn drive mass-loaded galactic winds and outflows to enrich the inter-galactic medium. In dwarf galaxies, the loss of material into the halo may be sufficient to reduce the apparent yield of the heavy elements. The formation of massive stars in the nuclear regions of galaxies may also assist the growth of the central Black Holes, by providing a source of viscosity to assist the circum-nuclear gas to shed its angular momentum. In turn, the jets from the resulting AGN can themselves trigger star formation during their passage through the galactic medium. Thus, massive stars truly act as "cosmic engines" exercising an important control on both the structural evolution of the Universe and on the structure and evolution of the galaxies which it contains.

There have been a number of excellent recent reviews covering particular aspects of the feedback process. The issue of massive star feedback on controlling the structure and porosity of the interstellar medium (ISM) through the formation of bubbles and super-bubbles has been reviewed by Oey \& Clarke (2008), see also Chu (this volume). A useful earlier review concerning the role of massive stars in controlling the morphology and physical structure of the ISM was published by Chu (1999). The physics of feedback processes such as accretion luminosity, ionizing radiation, and stellar winds in controlling the formation and growth of massive stars has been recently dealt with by Mac Low (2008). Various other aspects of feedback are covered in this conference. In particular I 
refer the reader to the paper by Sally Oey dealing with radiative feedback processes, Jay Gallager's report on feedback in starbursts, and both Yoshiaki Taniguchi's and Volker Bromm's reviews on the high redshift Universe and the first stars.

In this review, I will concentrate on the rapid progress which has been made in understanding feedback and its effects from a theoretical perspective. In particular, I will concentrate on our increasing understanding of the importance of feedback from the first stars in controlling the structural evolution of the Universe, and on the role of feedback in starburst environments.

\section{The Early Universe}

\subsection{The First Stars: An Overview}

For the issues associated with the formation of the first stars, we refer the reader to the extensive and fairly recent review by Bromm \& Larson (2004). The details of the problems associated with feedback from the first stars and its influence on the development of structure in the early universe has recently be reviewed by Ciardi (2008). She distinguishes between three types of feedback process - mechanical feedback which alters the phase structure and the distribution of gas following the formation of the first stars, chemical feedback which occurs once there are sufficient heavy elements to allow the cooling and fragmentation of dense clouds into stars with an initial mass function (IMF) similar to today's, and radiative feedback processes driven by the ionizing radiation of the massive stars. Since this review is so comprehensive and recent, we refer the interested reader to it, and here I will emphasize only a few very recent results.

The first Population III stars are probably described by a top-heavy IMF, a consequence of $\mathrm{H}_{2}$ cooling. Although it has been generally assumed that these formed at redshift $z \sim 20$ e.g. Yoshida et al. (2003), they may well have been formed as little as $\sim 30$ Myr after the Big Bang corresponding to $z \sim 65$; Naoz, Noter \& Barkana (2006). At this time the overdense regions contained as little as $10^{5} \mathrm{M}_{\odot}$. However, it is not until $\sim 500$ Myr after the Big Bang $(z \sim 12)$ that systems as large as the Milky Way start to assemble.

The formation of the Population III stars is strongly controlled by the formation and destruction of the $\mathrm{H}_{2}$ molecule, since this is the principle cooling agent. This physics and its consequences has been recently been discussed in detail by Mesinger, Bryan \& Haiman (2006). The strength of the UV radiation field is critical in controlling the $\mathrm{H}_{2}$ fraction, since molecular hydrogen is readily destroyed by photoabsorbtion into autoionizing states in the 11.2-13.6 eV Lyman-Werner Bands by the Solomon Process (Stecher \& Williams (1967), Abel et al. (1997)):

$$
\mathrm{H}_{2}+\gamma \rightarrow \mathrm{H}_{2}^{*} \rightarrow 2 \mathrm{H}
$$

Such a process provides a radiative negative feedback for star formation by suppressing cooling (Ciardi \& Ferrara (2005), Mesinger, Bryan \& Haiman (2006)). However, $\mathrm{H}_{2}$ formation is facilitated both by the availability of free electrons and by elevated temperature:

$$
\begin{aligned}
& \mathrm{H}+\mathrm{H}_{2}^{+} \rightarrow \mathrm{H}^{+}+\mathrm{H}_{2} \\
& \mathrm{H}+\mathrm{H}^{-} \rightarrow e^{-}+\mathrm{H}_{2} .
\end{aligned}
$$

Such conditions are found in regions shock-heated by colliding and merging filaments in the cosmic web, which therefore serve to facilitate the formation of the earliest stars. They are also to be found in cooling and recombination zones associated with fossil 
HII regions, in regions of X-ray heating by regions shock-excited by supernovae, i.e. regions surrounding these first stars during the time following the lifetime of the star as a powerful sources of UV radiation. In particular, Johnson, Greif \& Bromm (2007) find that the build up of $\mathrm{H}_{2}$ in the relic HII regions surrounding the first population of stars makes a large difference to the Lyman-Werner band radiative transfer. While the IGM is optically thick to Lyman-Werner photons over physical distances of $\sim 30 \mathrm{kpc}$ at redshifts $z \sim 20$, the high molecule fraction that is built up in relict H II regions as well as their increasing volume-filling factor renders their local IGM optically thick to Lyman-Werner photons over path lengths as short as a kiloparsec or so. For the halo population of stars, Mesinger, Bryan \& Haiman (2006) find that a Lyman-Werner background of as little as $J_{\mathrm{UV}} \sim 10^{-21} \mathrm{erg} \mathrm{s}^{-1} \mathrm{~cm}^{-2} \mathrm{~Hz}^{-1} \mathrm{sr}^{-1}$ may be sufficient to strongly inhibit star formation in the early universe. Similar conclusions are reached by Johnson, Greif \& Bromm (2007)

In the absence of a strong radiation field, the $\mathrm{H}_{2}$ fraction is correlated only with the virial temperature of the gas in any forming mass condensation. Yoshida et al. (2003b) find that the $\mathrm{H}_{2}$ fraction can be approximated by a power-law in terms of the virial temperature; $f_{\mathrm{H}_{2}} \propto T_{\text {vir }}^{1.52}$. In order to form cold clouds which can subsequently form the first Population III stars, the cooling timescale has to be shorter than the free-fall timescale. This then implies a mass boundary $\left(M \sim 7 \times 10^{5} \mathrm{M}_{\odot}\right)$ below which dark matter halos are unable to form cold clouds. These will subsequently lose their baryonic gas at the time of re-ionization, and will remain as dark satellites until they are incorporated into larger structures by merging.

\subsection{The Epoch of Re-ionization}

Re-ionization is an important phase transition in the history of the Universe, since it terminates the formation of the first stars and clusters in dwarf mini-haloes. Subsequently, star formation has to occur in much larger mass agglomerations as the first recognizable galaxies collapse and become self-shielding.

The process of re-ionization is driven by the first massive stars which formed in the Universe. The process of evolution of the first HII regions was treated in a 1-D fashion followed by a ray tracing methodology to trace the ionization of the surrounding medium by Alvarez, Bromm \& Shapiro (2006). They find, in contradiction to O'Shea et al. (2005) that that neighboring minihalos are not fully ionized before the initial star dies. The ionization rapidly moving ionization front gets trapped and converted into a D-type front outside the core region. Since the photoevaporation time for the minihalo exceeds the lifetime of the ionizing star, the core region remains shielded.

Recently, sophisticated 3-D radiative hydrodynamical models have been developed to attack this problem, first by Yoshida (2006) and Yoshida et al. (2007) and more recently by Wise \& Abel (2008a). These authors find that even single stars input sufficient energy to blow away and ionize much of the baryonic matter in their mini-halos. A consequence of this is that fairly large mini-halos with masses as large as $2 \times 10^{7} \mathrm{M}_{\odot}$ can be expected to be dark, see Read, Pontzen \& Viel (2006). The star initially ionizes a large region which first starts to expand from the local over-pressure before slowly recombining. The subsequent supernova evolves in the fossil HII region. Wise \& Abel (2008a) find that it is these SNR which exercise the greatest control on the re-ionization of the intergalactic medium, by increasing the porosity of this medium, and heating an appreciable fraction of the total volume to coronal temperatures. Subsequent generations of stars are then able to ionize a much larger region, since in many directions the absorption of the UV photons is low. Wise \& Abel (2008b) show that the voids also become highly metal enriched by this process, with the lowest density phases reaching close to solar metallicity at $z \sim 15$, while 
most of the denser phases are enriched with pair-instability supernova nucleosynthetic products only up to $\log Z / \mathrm{Z}_{\odot} \sim-3$.

The metallicity history of the Universe throughout cosmic time has been modeled by Kobayashi, Springel \& White (2007) using SPH methods of computation. This simulation is available on the web as a moviet. They also found that the enrichment history depends strongly on the environment. In large galaxies, enrichment takes place so quickly that $[\mathrm{O} / \mathrm{H}] \sim-1$ at $z \sim 7$, consistent with observations of Lyman break galaxies. The average metallicity of the universe reaches $[\mathrm{O} / \mathrm{H}] \sim-2$ and $[\mathrm{Fe} / \mathrm{H}] \sim-2.5$ at $z \sim 4(t=1.5 \mathrm{Gyr})$, but takes until $z \sim 3(t=2.1 \mathrm{Gyr})$ to reach this average value in the IGM. These models predict that the "metallicity floor" in the local universe would be of order $\log Z / Z_{\odot} \sim-2$.

To summarize the salient points of this section:

- The first stars form in dark matter halos having masses $\sim 10^{6} \mathrm{M}_{\odot}$ at $z \geqslant 18$.

- Weak UV fluxes stimulate both the formation of $\mathrm{H}_{2}$ and the rate of star formation in the Population III stars, but feedback becomes negative once the UV flux density exceeds a critical value.

- Radiation feedback expels the gas from the shallow potential wells of their dark matter halos. Below $\sim 2 \times 10^{7} \mathrm{M}_{\odot}$, galaxies will be dark, and in halos as large as $10^{8} \mathrm{M}_{\odot}$, as much as $75 \%$ of the baryons can be driven out into the intergalactic medium.

- The combination of radiation feedback and mechanical energy feedback from SNe creates a spatially complex ISM phase structure, with hot voids and a photoionized lowdensity phase which together occupy most of the volume. The lower density phases are rapidly enriched up to metallicities between $10^{-2}$ and $10^{-3}$ solar.

\section{Feedback at the Epoch of Massive Galaxy Formation}

The star formation history of the Universe is usually summarized in the form of a Madau - Lilly (or Lilly - Madau) plot, the star formation rate per unit co-moving volume of the Universe plotted against redshift (Lilly et al. 1996; Madau et al. 1996). Apart from the uncertainty introduced by the variety of heterogeneous samples and techniques, there is considerable uncertainty attached to the intrinsic reddening corrections, especially at high redshift. This said, it is remarkable that a fair degree of convergence is reached, with various methods agreeing within a factor of three or so. For a recent compilation from the literature, see Fardal et al. (2007), and for $z<0.5$, see Hanish et al. (2006).

From this plot, we can infer that there was a broad peak of star formation when the Universe was between 1 and 6 Gyr old. This was the epoch of massive galaxy assembly which gave rise to the passively-evolving "red-and-dead" Elliptical galaxies we see in the local volume. When we look back to this epoch of galaxy formation, we see these galaxies as infrared-bright, dusty galaxies detected either in the sub-mm region of the spectrum, the sub-mm galaxies (SMGs), or in the form of their radio-loud equivalents, the high-redshift radio galaxies (HizRGs)

For the SMGs Blain et al. (2004) has shown that, at a given dust temperature, the SMGs are typically 30 times as luminous as their ULIRG counterparts. Takagi et al (2003a, 2003b) had previously found that most ULIRGS have a constant surface brightness of order $10^{12} \mathrm{~L}_{\odot} \mathrm{kpc}^{-2}$. Our own results, Dopita et al. (2005), indicate that this corresponds to an ISM with a pressure of order $P / k \sim 10^{7} \mathrm{~cm}^{-3} \mathrm{~K}$. These parameters probably characterise "maximal" star formation, above which gas is blown out into the halo of the galaxy and star formation quenched. Only mergers, which provide an additional ram-pressure confinement of the star formation activity may exceed this surface

$\dagger$ see: http://th.nao.ac.jp/ chiaki/research-e.html 
brightness. Thus, in order to scale the star formation up to the rates inferred for SMGs $\left(\sim 1000-5000 \mathrm{M}_{\odot} \mathrm{yr}^{-1}\right)$, we must involve a greater area of the galaxy in star formation, rather than trying to cram more star formation into the same volume. For a typical value of $10^{13} \mathrm{~L}_{\odot} \mathrm{kpc}^{-2}$, we require "maximal" star formation over an area of $\sim 10 \mathrm{kpc}^{2}$, and the most luminous SMGs require star formation to be extended over an area of at order $\sim 100 \mathrm{kpc}^{2}$. We can conclude that SMGs are starbursts extended on a galaxy-wide scale, unlike the starburst regions seen in the nearby universe.

This violent galaxy-wide star formation epoch is terminated by the feeding and growth of the nuclear black hole which, when it grows beyond a critical mass is capable of expelling the gas from the host galaxies through the action of its relativistic jets.Evidence for this process comes from the study of the high redshift radio galaxies (Hi-zRGs) at $z>3$. These rank among the most luminous, largest, and most massive galaxies known in the early Universe (De Breuck et al. 2002) and which are observed early in the Epoch of Galaxy Formation at a time when their super-massive black holes (SMBHs) are highly active, and while their relativistic jets are interacting most strongly on their host galaxies. Spectroscopy by Reuland et al. (2007) has shown that the Lyman- $\alpha$ halos exhibit disturbed kinematics, with broad lines, large velocity shears, and expanding shells associated with the radio lobes. In addition, these halos halos are both chemically enriched by star formation and ionized throughout the majority of their volume. The relativistic jets drive strong shocks into the galactic medium and trigger shock-induced star formation on their peripheries.

These observations confirm the general physical idea of Silk \& Rees (1998) that it is the feedback of the black hole on its host galaxy which eventually limits the growth of the black hole. However, the observed feedback is not supplied by radiation pressure but rather by the mechanical energy input delivered by the relativistic jets. In the HizRGs we observe the moment where galaxy collapse gives way to mass ejection, and a newly born galaxy is revealed. We may further speculate that this is the defining moment where the such galaxies enjoy one last violent burst of shock-induced star formation before beginning their evolution to become the "red and dead" massive Elliptical galaxies we see in our local universe. This shock-induced star formation proceeds at several thousands of solar masses a year for at least a dynamical timescale, and is therefore capable of producing as much as $10^{11}-10^{12} \mathrm{M}_{\odot}$ of new stars. Stars formed by such shock interactions should have orbital characteristics quite unlike those of the earlier stellar generations. Traces of this epoch may therefore be found by a detailed study of the orbital dynamics of the massive elliptical galaxies in the local Universe.

\section{Starbursts \& Superwinds}

The basic physics of starburst-powered winds in galaxies is rather simple, and has been described by Chevalier \& Oegerle (1979). To power a wind, we require (at least) that the energy injection per unit area into a fraction, $f$, of the disk gas will exceed its binding energy. As Chevalier \& Oegerle (1979) has shown, this means that the gas escaping into the halo has an initial temperature greater than some critical value related to the binding energy. The physical parameters control the production of a galactic wind can be easily derived, The input energy $E_{\text {in }}$ per unit area is proportional to the surface rate of star formation $\dot{\Sigma}_{*}$ integrated over the lifetime of the starburst, $\tau_{\mathrm{SB}}$;

$$
E_{\mathrm{in}}=\alpha \dot{\Sigma}_{*} \tau_{\mathrm{SB}}
$$


The escape energy of the gas is

$$
E_{\mathrm{esc}}=0.5 f \Sigma_{\mathrm{gas}} v_{\mathrm{esc}}^{2}
$$

where $\Sigma_{\text {gas }}$ is the surface density of gas in the disk. The star formation rate is related to the gas surface density through the Kennicutt (1998) star formation law:

$$
\dot{\Sigma}_{*}=\beta \Sigma_{\text {gas }}^{1.5}
$$

Thus, a galactic wind becomes possible when:

$$
\frac{\tau_{\mathrm{SB}} \Sigma_{\mathrm{gas}}^{0.5}}{v_{\mathrm{esc}}^{2}} \geqslant \frac{f}{2 \alpha \beta} \sim \text { const. }
$$

Therefore, a wind is favoured when the escape velocity is low (such as will be the case in small galaxies), when the starburst is long continued (which is not surprising, since long lived star formation events can put in more energy to remove the gas), and (somewhat more surprisingly) when the gas surface density is high.

\subsection{Galactic Chimneys, Blowouts \& Fountains}

Our own galaxy is known to host a number of superbubbles, blowouts and chimneys. This had been demonstrated many years ago Heiles (1979a) and Heiles (1979b), but has been confirmed in exquisite detail by the high-resolution HI survey of McClure-Griffiths et al. (2002) and by her subsequent studies.

Two massive blow-outs are known in the Galaxy. McClure-Griffiths et al. (2003) have studied the Galactic chimney GSH $277+00+36$ in HI. This chimney is more than 600 pc in diameter, and extends at least $1 \mathrm{kpc}$ above and below the Galactic midplane. The shell and chimney walls exhibit a great deal of small-scale cold gas structures in HI in the form of loops or 'drips'. These exhibit narrow line widths in the range $1.5-2.5 \mathrm{~km}$ $\mathrm{s}^{-1}$. More recently, McClure-Griffiths et al. (2006) found a second example of a super bubble which has blown out of both sides of the galactic plane; GSH $242-03+37$. This has a radius $\sim 600 \mathrm{pc}$ and would require an input energy of $3 \times 10^{53}$ ergs to have imparted the kinetic energy of the expansion. Their HI images reveal a complicated shell with multiple chimney structures, capped by narrow filaments about $1.6 \mathrm{kpc}$ above and below the Galactic mid-plane.

The formation of these fine-scale HI structures remains a bit of a mystery, but a good deal of insight can be derived from the new 3-D radiative hydrodynamical computations made by Ralph Sutherland (in preparation), using his new code Fyris Alpha. He finds that breakout occurs in his simulations about $7 \mathrm{Myr}$ after the commencement of the starburst. The filamentary structure can be caused simply by the initial density fluctuations in the disk HI gas. Cooled HI droplets similar to those seen in the observations occur soon after breakout, and are visible up to the termination of the simulation at 25 Myr. Recombination of the denser material in the plane occurs after about $15 \mathrm{Myr}$, when the supernova energy input decreases. However, these later phases depend rather critically upon the lower mass limit for core-collapse supernovae, since the disappearance of supernova energy input terminates the phase in which the ejecta are being re-heated and driven into outflow.

The physics which drives these localized outflows is undoubtedly very similar to that which drives the high velocity low-intensity components seen in the ionized gas associated with supergiant HII regions in external galaxies. Relaño \& Beckman (2005) and Rozas et al. (2006) find that these are found in a substantial fraction of supergiant HII regions in nearby disk galaxies, and indicate expansion velocities of $100-160 \mathrm{~km} \mathrm{~s}^{-1}$ in the high velocity components of the $\mathrm{H} \alpha$-emitting gas. In Figure 1 we show the total kinetic energy 
( $\mathrm{H} \alpha$ line core plus the broad wings) we have derived for the Rozas et al. (2006) sample of supergiant HII regions, plotted against the mass of the exciting stellar cluster. This mass is derived from the $\mathrm{H} \alpha$ luminosity, assuming a Salpeter stellar Initial Mass Function (see Dopita et al. (2006)). As can be seen, at high cluster masses, the he kinetic energy content is proportional to the mass of the central stars (indicated by the solid line). This is in accordance with equation 4.1, above.

On a larger scale, Booth, Theuns \& Okamoto (2007) have used SPH simulations to provide the temporal evolution of disk galaxies based on a molecular cloud regulated model of star formation in the disk. For very young galaxies, a starburst-driven nuclear wind appears, but at later phases, a more generalized galactic wind is blown, with local hot-spots in the halo, and distinct dynamical structures in the wind. However, the resolution of these simulations in the halo regions is too low to give one full confidence in the results.

\subsection{Superwinds}

The poster child of Galactic winds is M82. However, it should always be recalled that M82 provides only a modest-sized starburst and associated galactic wind, hosted in a low-mass galactic system. McCarthy, van Breugel \& Heckman (1987) were the first to suggest that the starburst in this object was driving a wind, and this has since been abundantly confirmed by the exquisite HST, Spitzer and Chandra images e.g. Mutchler et al. (2007) which show a complex bipolar system of dusty $\mathrm{H} \alpha$ filaments being driven

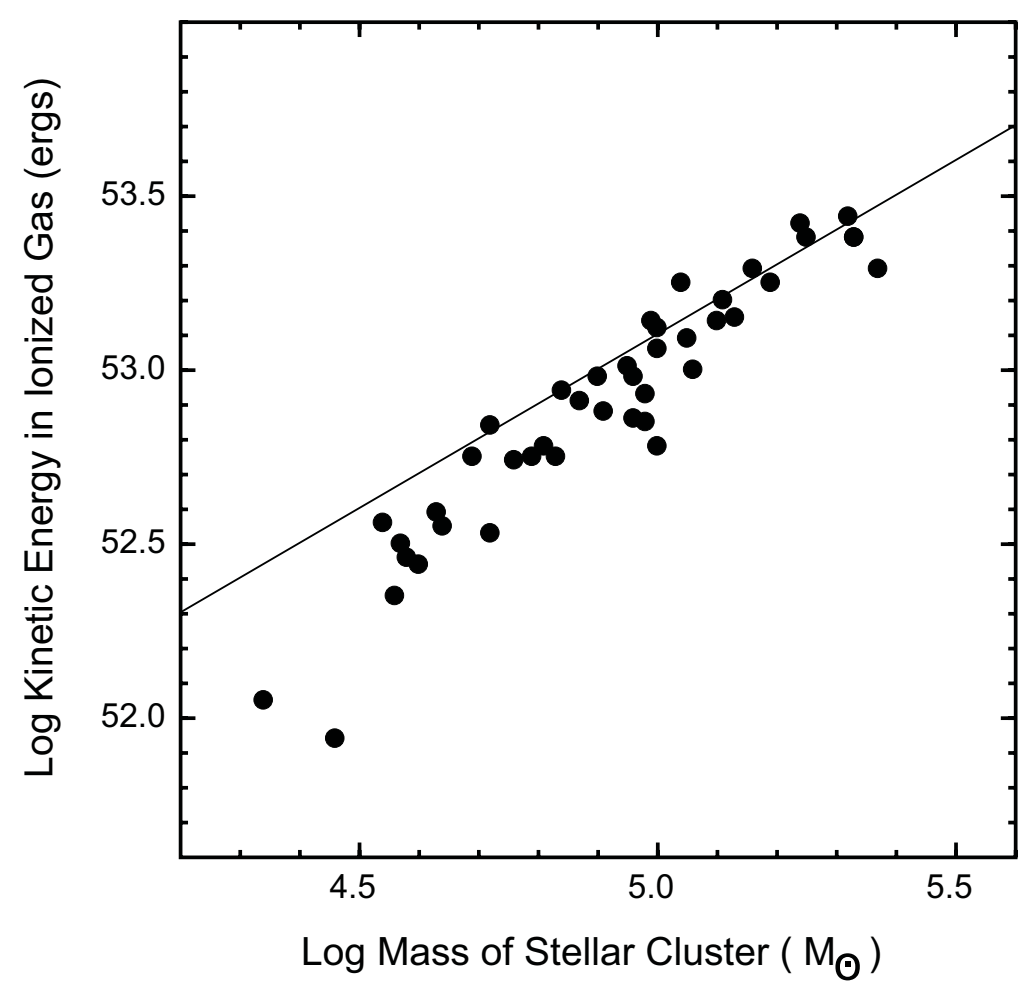

Figure 1. The kinetic energy content of the Rozas et al. (2006) HII regions as a function of the mass of the exciting stars. For the larger HII regions, the kinetic energy content is proportional to the mass of the central stars, in accordance with the expectation that the kinetic energy output of the stars drives both the turbulent and organized expansion velocities. 
out by a hot X-ray emitting plasma originating in the starburst core of this galaxy. Such bipolar winds are commonly found in galaxy interactions, e.g. in the SINGG survey Meurer et al. (2006), and can be important in polluting intergalactic space with the nucleosynthetic products of starbursts.

A large number of theoretical models have been constructed in the attempt to explain the structure of such starburst-driven winds, notably Tomisaka \& Ikeuchi (1988), Tomisaka \& Bregman (1993), Suchkov et al. (1994), Tenorio-Tagle \& Muños-Tuñòn (1998) and Strickland et al. (2000). Nearly all of these are 2-D simulations. Recently Cooper et al. (2008) (see also this volume) have made full radiative 3-D radiative hydrodynamical simulations which better capture the physical complexity of starburst-driven winds. They find that the hot gas escapes in channels between the denser and cooler gas that is itself entrained in the flow, eventually forming long radial filaments. The general features of this flow are shown in figure 2. The zones familiar from the theory of mass-loss driven bubbles e.g. Castor, McCray \& Weaver (1975) or Weaver et al. (1977) are clearly seen, although the bipolar structure and the entrainment zone at the base of the wind distinguishes these models from simple 1-D mass loss bubbles.

The limitation of the Cooper et al. (2008) and indeed, all other models is one of resolution. In order to properly resolve the cooling and entrainment regions a very high spatial resolution is required. Inadequate spatial resolution leads to over-cooling in the vicinity of the filaments, and an overestimate of the mass in the filaments. The highly non-spherical and non-uniform nature of the flow precludes the use of adaptive mesh

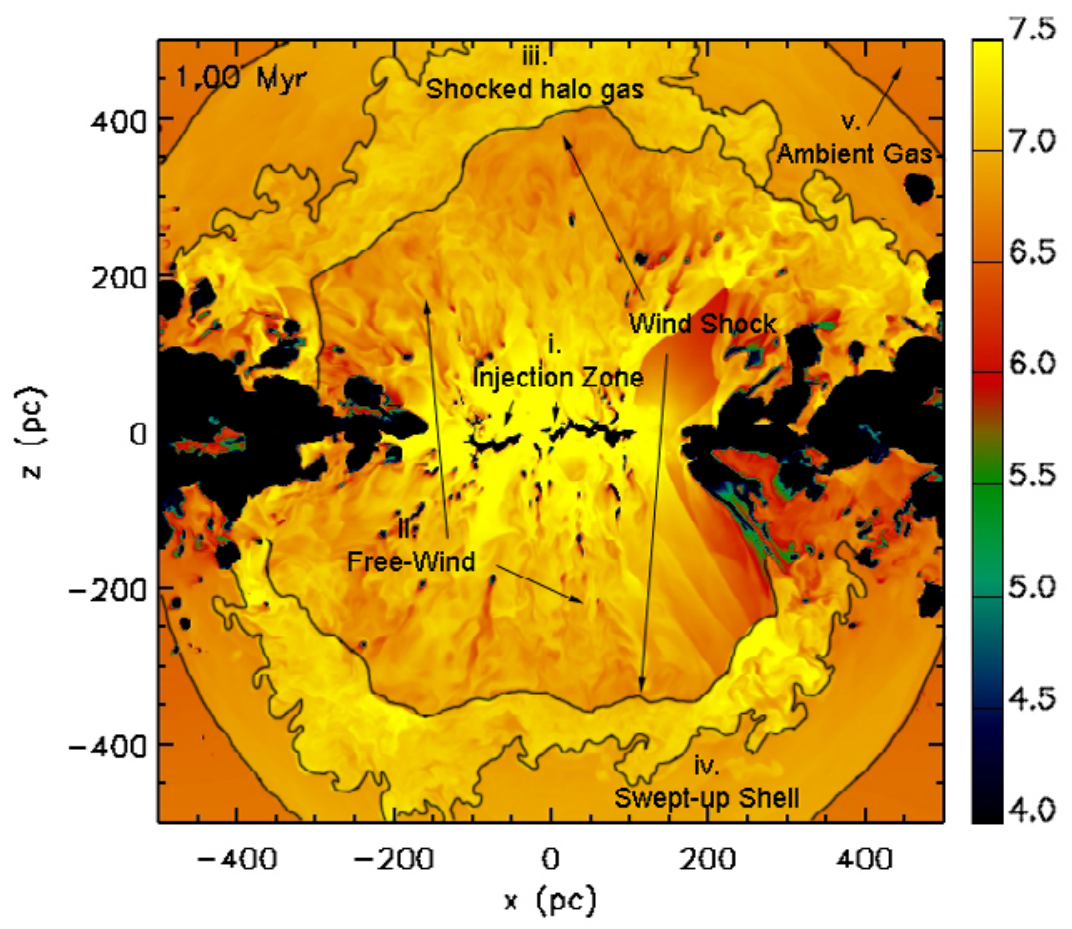

Figure 2. A slice through the 3-dimensional simulation of a galactic wind from Cooper et al. (2008), showing the various zones of the flow. The color coding gives the electron temperature of the plasma. 
techniques, so there is little choice other than using as large a grid as possible, and as large a supercomputer as is available.

Nonetheless, despite these limitations, the general features of the superwind displayed in the simulations are valid. In particular, the rapid outflow velocities predicted in the entrained cooled gas are observed in the luminous starburst galaxies. For example, Martin (2005) find that the $\mathrm{NaI} \lambda \lambda 5890,5896 \AA$ absorption lines indicate mean outflow velocities of over $300 \mathrm{~km} \mathrm{~s}^{-1}$ for the ultraluminous IR Galaxies (ULIRGs). She also infers that the rate of outflow of gas is comparable to the rate of consumption of gas through star formation, and that maximum outflow velocity is correlated with the star formation rate (SFR); $v \propto \mathrm{SFR}^{1 / 3}$. Even higher outflow velocities (between 490 and $2020 \mathrm{~km} \mathrm{~s}^{-1}$ ) have been found by Tremonti, Moustakas \& Diamond-Stanic (2007) in the Mg II $\lambda \lambda 2796,2803 \AA$ absorption lines associated with massive post-starburst galaxies as $z \sim 0.6$. These velocities are intermediate between ULIRGs and the Low-ionization Broad Absorption Line Quasars (LoBALs) Trump et al. (2006), suggesting that wind-driving by a buried AGN might be a rather significant contributor to the total kinetic energy budget of these objects.

\section{Conclusions}

In conclusion, it is fair to say that the confluence of high-quality spectrophotometric and imaging data obtained throughout the electromagnetic spectrum, coupled with theoretical results from the new generation of $3-\mathrm{D}$ radiative hydrodynamical codes is finally furnishing a coherent picture of starburst-powered galactic winds throughout cosmic time. The mass budget of these winds is a significant fraction of the total baryonic content of galaxies, and the winds provide much of the hot gas in galactic clusters and in the intra-cluster medium, as well as providing the source of the chemical enrichment of the intergalactic medium.

\section{References}

Abel, T., Anninos, P., Zhang, Y. \& Norman, M. L. 1997, New Ast., 2, 181

Alvarez, M. A., Bromm, V. \& Shapiro, P. R. 2006, ApJ, 639, 621

Blain, A. W., Chapman, S. C., Smail, I. \& Ivison, R. 2004, ApJ, 611, 52

Booth, C. M., Theuns, T. \& Okamoto, T. 2007, MNRAS, 376, 1588

Bromm, V. \& Larson, R. B. 2004, ARAA, 42, 79

Castor, J., McCray, R. \& Weaver, R. 1975, ApJ, 200, 107

Chevalier, R. A., \& Oegerle, W. R. 1979, ApJ, 277, 398

Chu, Y.-H. 1999, Ap\&SSS, 269, 441.

Ciardi, B. \& Ferrara, A. 2005, Space Sci. Rev., 116, 625

Ciardi, B. 2008, in: B. O'Shea, A. Heger \& T. Abel (eds.). First Stars III (New York: AIP) in press (arXiv:0709.1367)

Cooper, J. L., Bicknell, G. V., Sutherland, R. S., \& Bland-Hawthorn, J. 2008, ApJ, 674, 157

De Breuck, C., van Breugel, W., Stanford, S. A. et al. 2002, AJ, 123, 637

Dopita, M. A., Groves, B. A., Fischera, J. et al. 2005, ApJ, 619, 755

Dopita, M. A., Fischera, J., Sutherland, R. S. et al. 2006, ApJ, 647, 244

Fardal, M. A., Katz, N., Weinberg, D. H. \& Davé, R. 2007, MNRAS, 379, 985

Hanish, D. J. Meurer, G. R., Ferguson, H. C. et al. 2006, ApJ, 649, 150

Heiles, C. 1979, ApJ, 229, 533

Heiles, C. 1979, PASP, 91, 611

Johnson, J. L., Greif, T. H. \& Bromm, V. 2007, ApJ, 665, 85

Kennicutt, R. C. Jr. 1998, ApJ, 498, 541

Kobayashi, C., Springel, V. \& White, S. M. 2007, MNRAS, 376, 1465 
Lilly, S. J., Le Fevre, O., Hammer, F. \& Crampton, D. 1996, ApJL, 460, L1

McCarthy, P. J.; van Breugel, W. \& Heckman, Ti. 1987, AJ, 93, 264

McClure-Griffiths, N. M., Dickey, J. M., Gaensler, B. M. \& Green, A. J. 2002, ApJ, 578, 176

McClure-Griffiths, N. M., Dickey, J. M., Gaensler, B. M. \& Green, A. J. 2003, ApJ, 594, 833

McClure-Griffiths, N. M. Ford, A., Pisano, D. J. et al. 2006, ApJ, 638, 196

Mac Low, M.-M. 2008, in: H. Beuther et al. (eds.), Massive Star Formation: Observations Confront Theory (San Francisco: ASP), ASP Conf Ser. in press (arXiv:0711.4071)

Madau, P., Ferguson, H. C., Dickinson, M. E. et al. 1996, MNRAS, 283, 138

Martin, C. 2005, ApJ, 621, 227

Mesinger, A.,Bryan, G. L. \& Haiman, Z. 2006, ApJ, 648, 835

Meurer, G. R., Hanish, D. J., Ferguson, H. C. et al. 2006, ApJS, 165, 307

Mutchler, M. Bond, H. E., Christian, C. A. et al. . 2007, PASP, 119, 1

Naoz, S., Noter, S. \& Barkana, R. 2006, MNRAS, 373, 98

OShea, B. W., Abel, T., Whalen, D., \& Norman, M. L. 2005, ApJ, 628, L5

Oey, M. S. \& Clarke, C. J. 2008, in: M. Livio (ed.), Massive Stars: From Pop III and GRBs to the Milky Way, (Cambridge: CUP) in press (astro-ph/0703036)

Read, J. I, Pontzen, A P. \& Viel, M. 2006, MNRAS, 371, 885

Relaño, M., \& Beckman, J. E. 2005, A\&A, 430, 911

Reuland, M. van Breugel, W., de Vries, W. et al. 2007, AJ, 133, 2607

Rozas, M., Richter, M. G., López, J. A., et al. 2006, A\&A, 455, 549

Silk, J. Rees, M. J. 1998, AESA, 331, L1

Stecher T. P, Williams D. A. 1967, ApJ, 149, L29

Strickland, D. K., Heckman, T. M, Weaver, K. A. \& Dahlem, M. 2000, AJ, 120, 2965

Suchkov, A. A., Balsara, D. S., Heckman, T. M. \& Leitherer, C. 1994, ApJ, 430, 511

Tenorio-Tagle, G. \& Muños-Tuñòn, C. 1998, MNRAS, 293, 299

Tremonti, C. A., Moustakas, J. \& Diamond-Stanic, A. M. 2007, ApJ, 663, L77

Trump, J. R., Hall, P. B., Reichard, T. A. et al. 2006, ApJS, 165, 1

Tomisaka, K. \& Ikeuchi, S. 1988, ApJ, 330, 695

Tomisaka, K. \& Bregman, J. N. 1993, PASJ, 45, 513

Weaver, R., McCray, R., Castor, J., Shapiro, P. \& Moore, R. 1977, ApJ, 218, 377

Wise, J. H. \& Abel, T. 2008a, ApJ, in press (arXiv:0710.4328)

Wise, J. H. \& Abel, T. 2008b, ApJ, in press (arXiv:0710.3160)

Yoshida, N., Sokasian, A., Henquist, L. \& Springel, V. 2003a, ApJ, 598, 73

Yoshida N, Abel T, Hernquist L, Sugiyama N. 2003b, ApJ, 592, 645

Yoshida, N. 2006, New Ast. Revs., 50, 19

Yoshida, N., Oh, S. P., Kitayama, T., \& Hernquist, L. 2007, ApJ, 663, 687

\section{Discussion}

STANEK: We have evidence that the Mg II "clouds" at $z \sim 2$ have small sizes, which would indicate that they are short-lived. Do you think that these super-winds could give rise to the $\mathrm{Mg}$ II complexes seen in QSO and GRB spectra?

DopITA: This could well be. Crystal Martin, for example, finds outflows of roughly 300 $\mathrm{km} \mathrm{s}^{-1}$ in absorption in the high-z ULIRGS she has looked at. See ApJ 621, 227 (2005). The mass loaded outflows we model certainly are fragile, and rather transitory objects. The GRBs seem so be associated with low-mass galaxies, so outflows can be driven with quite modest rates of star formation.

STANEK: What is the fraction of total star formation that is in these starburst galaxies?

DopiTA: In the local universe, the fraction is rather small. However, by $z \sim 2$, the star formation is dominated by starburst galaxies. 
SonneBorn: Do the galactic fountain models you describe produce a hot $T>3 \times 10^{5} \mathrm{~K}$ component in the outflowing gas?

DopITA: Yes. These models are powered by the energy injection of shocked stellar winds and supernova explosions. The outflowing medium definitely forms a two-phase medium with one phase at coronal temperatures. However the final temperature in this phase depends on the details of the $P d V$ work done in escaping the potential of the galaxy and on the degree of mixing and mass entrainment that has occurred.

KUDRITZKI: You referred to the simulation of Kobayashi, which showed the the IGM was very quickly enriched to $\log \left[Z / \mathrm{Z}_{\odot}\right] \sim-2$ at high redshift. If this is true, how does one explain the existence of very metal poor stars in the Galactic Halo?

DopITA: The ISM is really quite poorly mixed, and the IGM enrichment occurs in the low density phase. In the simulations of Wise \& Abel (2007), the dense phase of the ISM is enriched only up to $\log Z / \mathrm{Z}_{\odot} \sim-3$ while the lowest density phases are enriched up to solar metallicity. Thus, IGM enrichment does not preclude the formation of low metallicity stars. It is more relevant in relation to our efforts to determine the metallicity floor of the local universe, since this metal enriched IGM gas is likely to have only recently been captured by dwarf star forming galaxies in the Local Universe.

ZINNECKER: Can you give us a general definition of a "starburst" i.e. in terms of star formation rate, per unit area, per unit time or what?

DopITA: The term "starburst" has been much used and abused, and there is currently no generally accepted definition. However, personally I would emphasize the importance of concentration, in terms of star formation per unit area, gas surface density or pressure in the star forming region. I suggest that a good working definition would be that $\log P / k \gtrsim$ $6 \mathrm{~cm}^{-3} \mathrm{~K}$ in the star-forming region. Star formation regions characterized by such high pressures in the galactic plane would then stand a good chance to be able to blow gas out into their galactic haloes as a galactic wind.

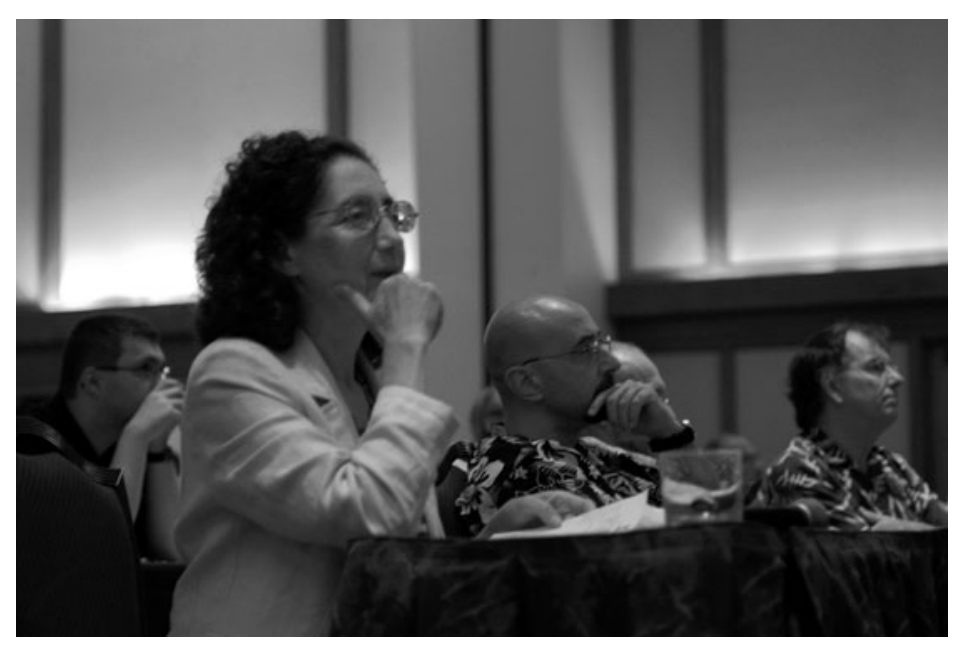

Gloria Koenigsberger (left), Marco Limongi (center) and Mike Dopita (right). 


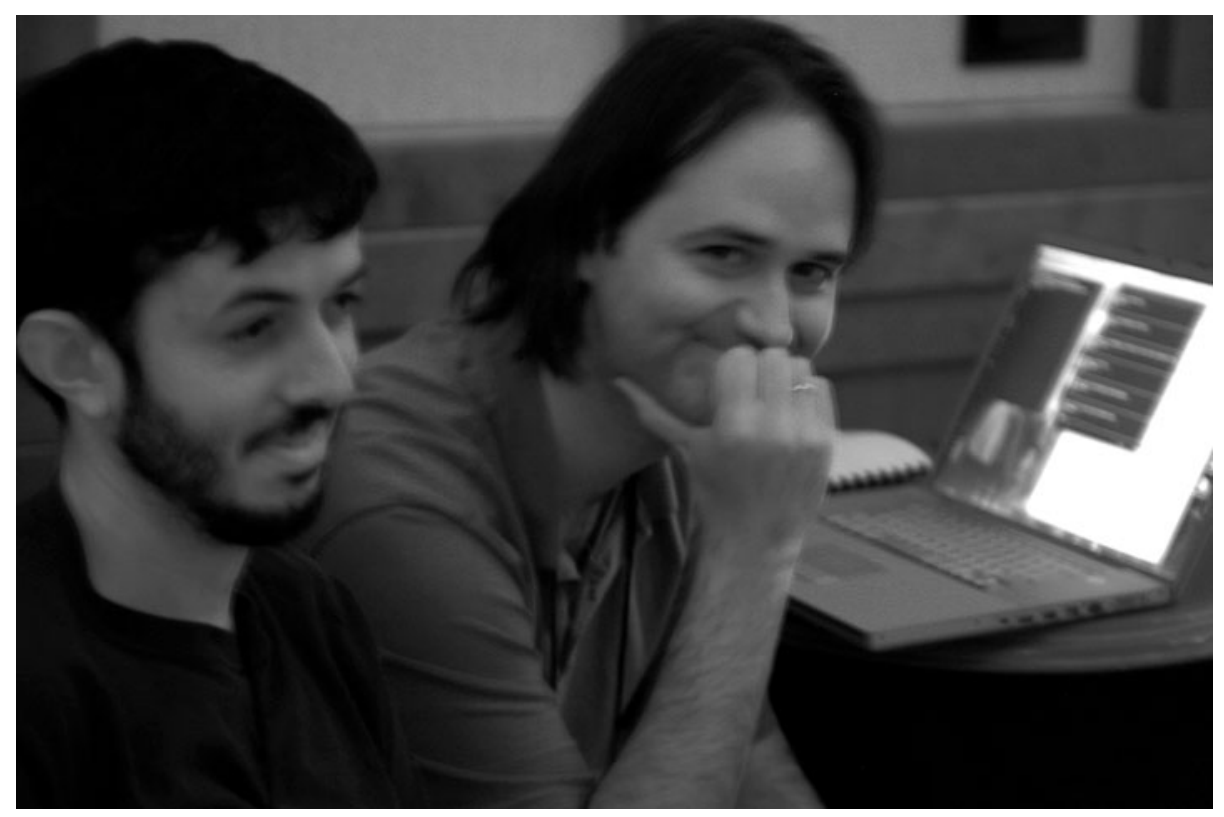

Alfredo Sota (left) and Jesús Maíz Apellániz (right).

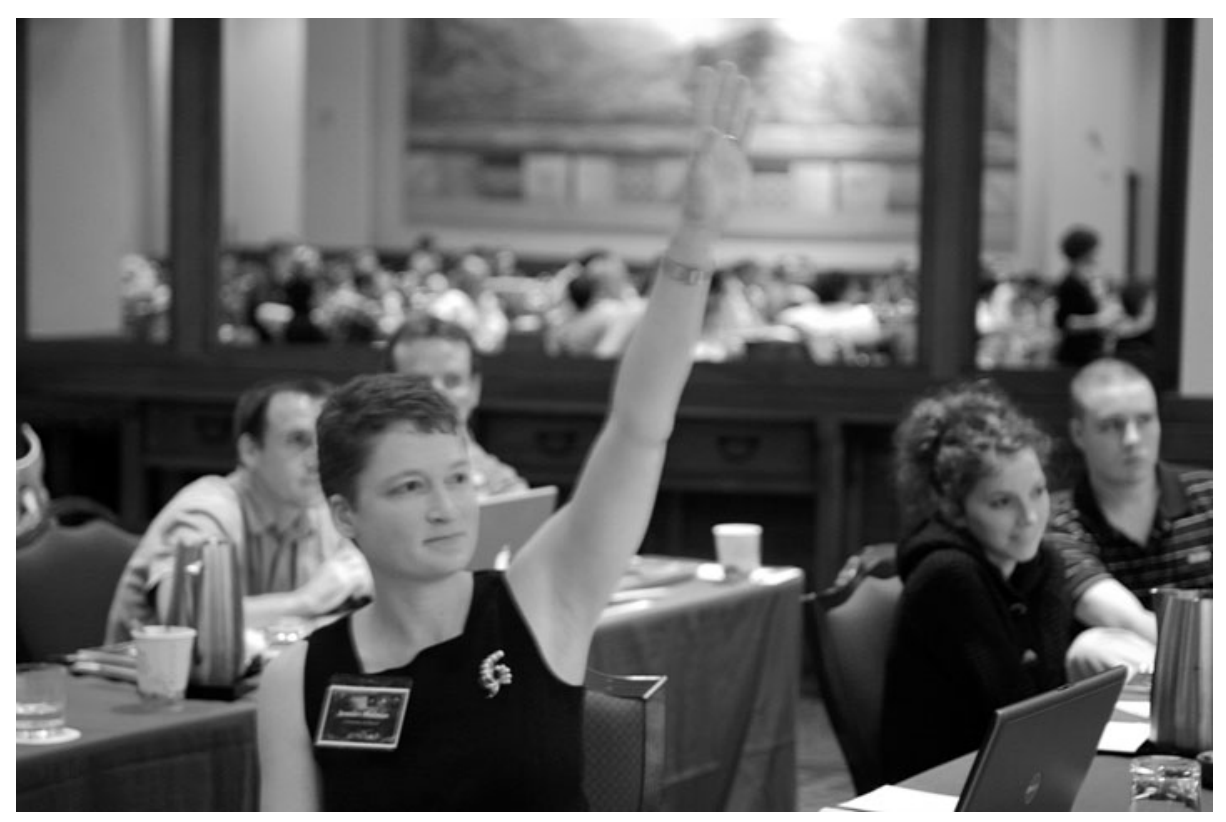

Jennifer Hoffman. 